\title{
Effects of change in smoking habits on bladder cancer incidence in Tunisia
}

\author{
Dhafer Mrizak $^{1}$, Fatma B'chir ${ }^{1,2}$, Mehdi Jaidane ${ }^{3}$, Maurice Jean Arnaud ${ }^{4}$, Saâd Saguem ${ }^{\text {* }}$ \\ ${ }^{1}$ Metabolic Biophysics and Applied Pharmacology Laboratory, Department of Biophysics, Medicine Faculty of Sousse, Sousse, \\ Tunisia; *Corresponding Author: bchirfatma@hotmail.fr \\ ${ }^{2}$ Laboratory of Natural Substances, National Institute of Research and Physical Chemical Analysis, Technopole Sidi Thabet, Tunisia; \\ ${ }^{3}$ Urolology Department of Central Hospital University (CHU), Sahloul-Sousse, Tunisia; \\ ${ }^{4}$ Nutrition \& Biochemistry, Bourg Dessous 2A, La Tour de Peilz, Switzerland.
}

Received 22 January 2011; revised 19 February 2011; accepted 28, February 2011.

\begin{abstract}
Bladder cancer is among the most frequently diagnosed cancer. Tobacco smoking exposures involving nitrosamines and aromatic amines are the main cause of bladder cancer. Although cigarette consumption has gradually decreased, an increased incidence of bladder cancer was registered among males Tunisian for the last two decades. A similar increased incidence of lung adenocarcinoma and bladder cancer was registered among Tunisian males. Epidemiological results suggest that changes in cigarette smoking may be the cause of the increased incidence of bladder cancer. The relationship between CYP1A2 enzyme activities, a key enzyme for activation of bladder carcinogens, and bladder cancer risk was investigated. Variations in CYP1 A2 activities measured in patients with bladder cancer and healthy smokers showed a signifycantly higher CYP1A2 metabolic activity in patient group. Changing in cigarette smoking habits associated to the variation in CYP1A2 activity seem to explain partly the increase incidence of bladder cancer observed in Tunisian male population.
\end{abstract}

Keywords: Cigarette Filter; Smoking;

Bladder Cancer; CYP1A2 Activity

\section{INTRODUCTION}

Bladder cancer is the most frequent urogenital cancer in Tunisia and rank at the second position in the world [1], among male populations.

Epidemiologic and biological studies have clearly established that cigarette smoking is the main cause of bladder cancer $[2,3]$ and it was suggested that more than $60 \%$ of all bladder cancer cases may be attributed to tobacco smoking [4,5].

Although stabilization and even decrease frequencies of major cancers types which are associated to smoking such as lung cancer and oral cavity cancer, a dramatic increase in the incidence of bladder cancer was registered in Tunisian Cancer Centers for the last two decades. This increasing prevalence has been also reported worldwide [6,7] and may be the result of changes in smoking behavior in particular when the cigarette with filter had replaced those with no filter.

The concentrations of specific carcinogens such as tobacco-specific nitrosamines (TSNs) and Aromatic Amines (AA) have been increased in filtered cigarette smoke [8].

It has been suggested that the consumption of those cigarettes with filters may contribute to increase bladder exposure to tobacco carcinogens thus leading to a higher risk of cancer development [8-10].

The aim of this study was to evaluate from epidemiological and biological data whether the introduction of cigarette with filter could explain the remarkable increase of bladder cancer incidence observed in Tunisian population.

\section{MATERIALS \& METHODS}

\subsection{Epidemiological Study}

Data of epidemiological study were obtained from CHU Central Tunisian hospital registers (Department of Anatomy Pathology of CHU Farhat Hached of Sousse, Tunisia) for the 1987-2005 time period and the evolution of bladder cancer incidence in Tunisian males was evaluated.

\subsection{Biological Study}

This study was carried out at the Faculty of Medicine of Sousse by the Biophysics laboratory where the assessment of CYP1A2 enzyme activity was measured by 
caffeine test among bladder cancer and healthy smoker's subjects.

CYP1A2 activity was estimated in vivo using caffeine as a probe drug and reversed-phase HPLC method was developed to measure plasma caffeine and paraxanthine concentrations to determine the CYP1A2 activity from the ratio paraxanthine/caffeine.

\subsection{Subjects}

14 patients with bladder cancer and 14 healthy subjects were recruited for this study.

All subjects are current smokers. They gave their informed consent and the study protocol was approved by the local Ethical Committee.

All subject abstained to drink any beverages containing caffeine for more than 24 hours before and during the study.

\subsection{Methods}

The subjects were asked to fill a questionnaire where the total consumption of coffee and other caffeine-containing beverages as well as the number daily cigarettes smoked and the period of smoking were recorded (Table 1).

The subjects were coming at the hospital in the morning at $8 \mathrm{am}$ and ingested $140 \mathrm{mg}$ of caffeine dissolved in $150 \mathrm{ml}$ warm water. A single $5 \mathrm{ml}$ blood sample was collected directly in EDTA tube four hours after caffeine ingestion.

Blood samples were immediately centrifuged at 3000 rpm for 15 minutes and plasma samples collected were stored at $-20^{\circ} \mathrm{C}$ until analyzed.

\subsection{HPLC Plasma Analysis and Caffeine Concentrations}

To $0.5 \mathrm{ml}$ defrost plasma was added $300 \mathrm{mg}$ of ammonium sulfate and after 1 minute vigorous shaking (Vortex), $3 \mathrm{ml}$ of ethyl acetate and isopropyl alcohol (8/2, v/v) were added. After shaking for 2 minutes, the samples were centrifuged at $4000 \mathrm{rpm}$ for 25 minutes and $2.5 \mathrm{ml}$ of the organic phases were collected, dried at $56^{\circ} \mathrm{C}$ under a flow of nitrogen gas and the residue obtained was dissolved in $100 \mu \mathrm{KH}_{2} \mathrm{PO}_{4}$ buffer solution.

Table 1. Averages daily cigarettes smoked and smoking period in bladder cancer patients and healthy controls.

\begin{tabular}{ccc}
\hline Groups & $\begin{array}{c}\text { Bladder cancer } \\
\text { patients }\end{array}$ & Healthy control \\
\hline Daily cigarettes smoked & 25 & 20 \\
Smoking period (years) & $43.5^{*}$ & 26.78 \\
\hline$* \mathrm{P}<0.05$ & & \\
\end{tabular}

HPLC (Agilent 1200) separation was performed using two reversed phase columns in series (Hypersil ODS $4 \times$ $250 \mathrm{~mm}, 5 \mu \mathrm{m}$ ) and (Eclipse XDB-C18 $4.6 \times 150 \mathrm{~mm}, 5$ $\mu \mathrm{m})$ maintained at $33^{\circ} \mathrm{C}$ with a direct injection of $5 \mu \mathrm{l}$ of the extract. An U.V diode array was used for the analytic detection. Elution was performed by gradient mode using two mobiles phases A and B. Phase A solution was composed of water, tetrahydrofurane (THF), acetic acid and acetonitrile (986/3/1/10 v/v respectively). The phase $\mathrm{B}$ solution was acetonitrile. The gradient program was chosen as follows: B was set at $0 \%$ for the 20 first minutes, increased linearly to $4 \%$ at 35 minutes, reached to $8 \%$ from 45 to 50 minutes and return to $0 \%$ at 55 minutes.

Plasma caffeine and the concentrations of its metabolites were calculated by CHEMSTATION software using calibrations curves already established.

\subsection{Measurement of Caffeine Metabolic Ratio}

Caffeine is mainly metabolized by the hepatic CYP1A2 enzyme and is currently used as a probe for the measurement of CYP1A2 activity. In the metabolism of caffeine, the first demethylation steps gave paraxanthine (PX, 17X), theobromine (TB, 37X) and theophylline (TP, 13X). About $80 \%$ of the metabolism of caffeine is going through the paraxanthine pathway mediated by CYP1A2 [11].

The measurement of the concentration ratio $\mathrm{PX}$ to caffeine (PX/CA) was used as a metabolic marker to evaluate the CYP1A2 activity.

\subsection{Statistical Analysis}

Mann-Whitney U test was used to examine differences in distributions of smoking period and caffeine metabolic ratio between cases and controls.

\section{RESULTS}

\subsection{Epidemiological Study}

\subsubsection{Evolution of the Incidence of Tobacco Related Cancers in Tunisia}

This study recorded the most frequent cancer associated to tobacco use in the Tunisian male population: larynx, oral cavity, bladder and lung.

The frequencies of these four cancers obtained from CHU Cancer register of Sousse are calculated for two periods 1987-1990 (PI) and 2002-2005 (PII). In Figure $\mathbf{1}$ is shown the evolution of the incidence curves of lung, bladder, oral cavity and larynx cancers for the time period 1987-2005.

Although stabilization and even a small decline in the 


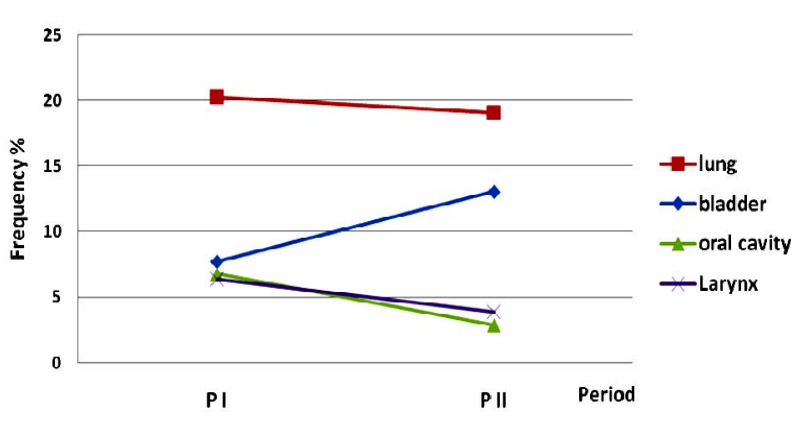

Figure 1. Incidence evolution of four tobacco-related cancers in Tunisia between the time periods PI (1987-1990) and PII (2002-2005).

incidence was observed for lung, larynx and oral cavity cancers, an increase of the incidence of bladder cancer was observed for the 2002-2005 period when compared to the 1987-1990 period. Bladder cancer frequencies exhibited increase from $7.69 \%$ in 1990 to $13.03 \%$ in 2005 and thus double in 15 years.

\subsubsection{Evolution of Lung Cancer and Its Histological Forms Incidence in Tunisian Population}

According to our previous study [12], the distribution of lung cancer incidence compared to its major histological types the adenocarcinoma (AD) and the squamous cell carcinoma (SCC) was not similar for the last two decades in Tunisian population.

As shown in Figure 2 a slow decline in the incidence for both total lung cancer and SCC was observed from 1990 up to 2005 period, while a continuous increase was registered for $\mathrm{AD}$ incidence.

\subsubsection{Comparative Analysis of the Bladder Cancer and AD Frequencies Evolution}

In Figure 3 is shown the evolution of the incidence of $\mathrm{AD}$ and bladder cancer for the 1987-2005 period. The evolution of both frequencies of bladder cancer and $\mathrm{AD}$ were similar during the same period of time. Bladder cancer and $\mathrm{AD}$ have gradually and simultaneous increased for these last two decades in Tunisian males.

\subsection{Biological Study}

\subsubsection{Separation of Caffeine and Its Metabolites}

The Figure 4 illustrates the chromatographic profiles of caffeine and its metabolites measured at $280 \mathrm{~nm}$ in plasmatic sample of one patient and one healthy subject. The chromatogram shows a complete separation of caffeine and its metabolites, in particular for TP, TB and PX. This good separation allows an accurate quantification of caffeine metabolites concentrations and consequently

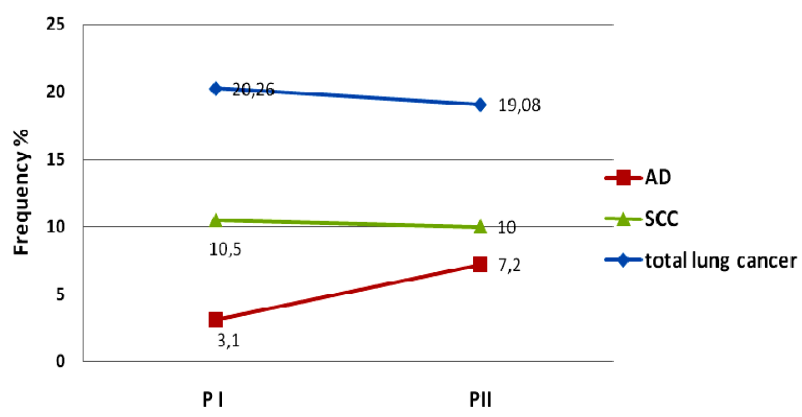

Figure 2. Incidence evolution of $\mathrm{AD}, \mathrm{SCC}$ and lung cancer in Tunisia between the time periods PI (1987-1990) and PII (2002-2005).

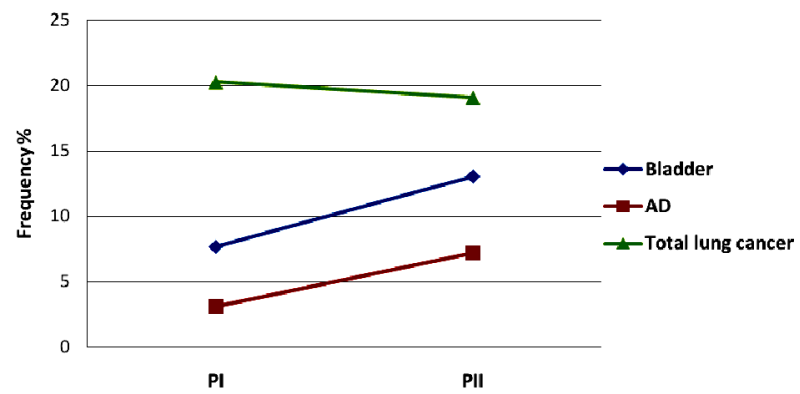

Figure 3. Evolution profile of $\mathrm{AD}$ and bladder cancer for the 1987-2005 period.

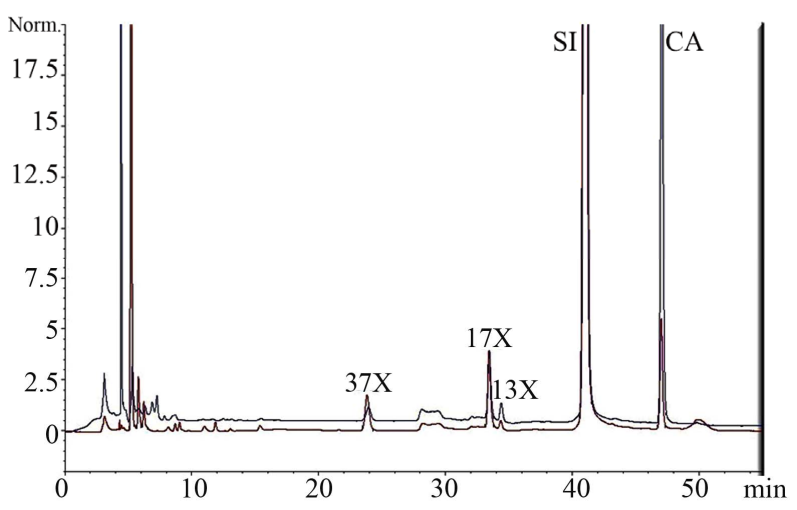

37X: 3,7 dimethylxanthine (theobromine); 17X: 1,7-dimethylxanthine (paraxanthine); 13X: 1,3-diméthylxanthine (theophylline), SI: Internal Standard; CA: 1,3,7-trimethylxanthine (caffeine).

Figure 4. Chromatograms of plasma samples of caffeine and its metabolites among one patient (blue) and one control subject (red).

the determination of the PX/CA metabolic ratios. The chromatographic profiles of plasma show that there was no interference with endogenous compounds.

\subsubsection{CYP1A2 Activity Evaluation}

Variations in PX/CA ratio were observed in Figure 5 between patients and healthy subjects. These variations suggest wide interindividual differences in CYP1A2 activities. The average PX/CA ratio was 0.42 for the bladder 


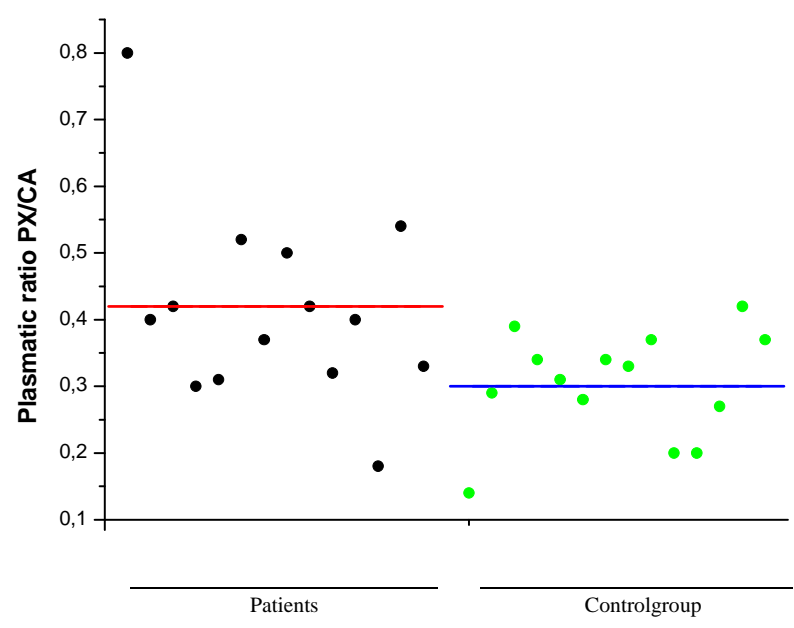

Figure 5. Distribution of PX/CA plasmatic ratio values among patients and control group.

cancer group and 0.3 for healthy group of subjects. The CYP1A2 activity was thus significant higher in the bladder cancer patient group compared to healthy group $(\mathrm{P}<0.05)$.

\section{DISCUSSION}

In the present study, biological and epidemiological investigations show that the rapid increase in the incidence of bladder cancer observed for the last two decades in Tunisian population may be related to the change in smoking behavior when the cigarettes with filter had substituted progressively those with no filter.

More than 50 years ago, tobacco use was suggested as a major etiologic risk factor for bladder cancer $[8,13]$ and $60 \%$ was attributed to cigarette smoking [4]. Many case-control studies reported that smokers have at least a two-fold higher risk of developing bladder cancer compared to non smokers $[8,14]$. Bladder cancer is estimated as the second cancer localization attributed to smoking after the lung [15].

The epidemiological results of this study show an opposite evolution of bladder and total lung cancer frequencies for the time period 1987-2005. Despite the stability of lung cancer incidence in Tunisian males for the 1987-2005 period (Figure 3), a two-fold increased bladder cancer frequency was registered from $7.69 \%$ in 1990 to $13.06 \%$ in 2005. This dramatic increase of bladder cancer incidence registered in Tunisia was also reported all over the world during the same time period. Cancer registers show a larger increase in Denmark, in USA and in France among men and a somewhat smaller increase among women [16,17]. This incidence is still increasing overtime. It has been suggested that heavy cigarette smoking plays a major role in increasing bladder cancer incidence [18]. This conclusion was also con- firmed in our work. Patients with bladder cancer are heavier smokers that subjects in the control group (Table 1).

How can be explained the apparent disagreement between the increase incidence of bladder cancer and the decline of the lung cancer frequency with a change in smoking behavior. We suggested that the changes in smoking behavior associated to the increased use of cigarettes with filter may explain the changes observed [12]. In this study was reported a stabilization of the total incidence of lung cancer while a continuous and significant increase of AD over SCC forms was observed among Tunisian population for the time-period 1987-2005.

Figure 6 shows that the $10.5 \%$ incidence of SCC is unchanged while that of $\mathrm{AD}$ exhibits an increase from $3.1 \%$ in 1990 to $7.2 \%$ in 2005 . This increase of AD was related to the introduction of cigarette with filter [12]. This new smoking habit appeared to be more carcinogenic through the increasing incidence of $\mathrm{AD}$, the most aggressive and metastasis cell types of lung carcinoma [19]. The smoker of cigarette with filter is more exposed to tobacco nitrosamines (TSNs) in particular the 4-methylnitrosamino-1-3-pyridyl-1-butanone (NNK); a potent carcinogen which induce lung tumors in the peripheral lung regions, the areas where the $\mathrm{AD}$ occurs $[12,20]$.

The significant increase of bladder cancer observed during the 1987-2005 time-period parallels the evolution of AD incidence in Tunisian population suggesting that both pathologies may have the same origin. The most prominent change in smoking habit reported during this period was the increased use of cigarettes with filter. Filter cigarette deliver at least $60 \%$ less nicotine and tar than plain cigarette $[5,8]$. While this change leads to a lower exposure of polycyclic aromatic hydrocarbons (PAHs), the concentrations of aromatic amine increased by 59\% from 1968 to 1985 [8] and between 1978 and 1995, the concentrations of tobacco-specific nitrosmines in filtered cigarettes increased $17 \%$ for N'-nitrosonornicotine and $44 \%$ for 4 -(methylnitrosamino)-1-(3-pyridyl)1-butanone [8,21]. Some N-nitroso-compounds such NNK have been suggested to be involved as causative agents in human lung carcinogenesis leading to the AD type $[20,22]$.

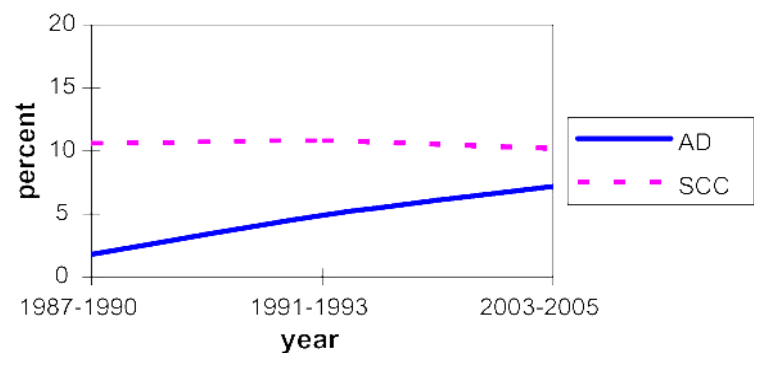

Figure 6. Trends of the evolution of $\mathrm{AD}$ and SCC in Tunisia. 
Moreover, the smoker's dependence on nicotine may lead to smoking more cigarettes with filter because they contain lower amounts of nicotine. To achieve the desired physiological response to nicotine, smokers of lowtar filter cigarettes tend to adapt their smoking patterns by taking larger puffs, increasing puff frequency and inhaling more deeply. Such changing smoking patterns showed that although the smoker-weighted carbon monoxide-yield of ventilated filter cigarettes was $21 \%$ less than that of plain cigarettes, blood carboxyhemoglobin level in ventilated filter cigarettes was $7 \%$ higher than those found in men who smoked plain cigarettes [23]. These data are consistent with the conclusion that smokers who are addicted to low-nicotine cigarettes may take more intense smoking and deeper inhalation of the smoke leading to a higher amount of carcinogenic tar particles, in the bronchi and bladder of the smoker [24].

Although the increasing incidences of $\mathrm{AD}$ and bladder cancer may have the same origin with the use of cigarette filter, they have different carcinogenic inducers. PAHs such as benzo $(a)$ pyrene $(\mathrm{BaP})$ and TSNs like NNK are the most known tobacco smoke potent carcinogens as shown on animal model $[25,26]$. PAHs and NNK cause mainly lung cancers, PAHs induce the SCC tumours occurring in bronchi and bronchioles of the lungs and NNK induce AD predominately in the deeper region of the lung. In animals exposed to the mainstream cigarettes smoke develop bladder cancer while animals treated with BaP or NNK develop cancers on other localizations than the bladder [27]. Thus, the induction of bladder tumours by tobacco substances inhaled is not dependent upon PAHs and TSNs, but may dependent on other tobacco chemicals $[18,28]$. It was reported that aromatic amines are potent bladder carcinogens [29]. Bladder tumours induced in animals with tobacco aromatic amines carcinogens [30] showed that these compounds do not elicit directly bladder tumours but the effects were due to some of their metabolites. Among these tobacco amines carcinogens, 2-naphthylamine and 4-aminobiphenyl were found to be the most potent bladder carcinogens in man [31]. The most direct-acting carcinogens that are reactive electrophilic agents are produced through in vivo activation involving an initial oxidation catalyzed primarily by hepatic cytochromes P450 [31], the first critical step in this bio activation [32, 33]. 2-Naphthylamine and the 4-aminobiphenyl are metabolized mainly in liver to N-hydroxy-4-naphthylamine (N-OH-naphthylamine) and $\mathrm{N}-\mathrm{OH}$-aminobiphenyl respectively. These reactive metabolites produce human epithelium DNA adducts in liver and bladder. The enzyme primarily responsible for the N-hydroxylation of 2naphthylamine and the 4-aminobiphenyl, the initial step for carcinogenic process, is the CYP1A2 [31]. This key enzyme in the activation of bladder cancer carcinogens exhibits wide variations in activity explaining individual susceptibility to chemical carcinogenesis and thus to bladder cancer development.

In smokers, the results of this study show a significant higher CYP1A2 activity in patients with bladder cancer when compared with healthy subjects. High CYP1A2 activity is therefore suggested as a susceptibility factor of bladder cancer. The polymorphic CYP1A2 activity may be related to environment and genetic factors. Exposure to tobacco smoke is known as a potent inducer of CYP1A2 activity $[34,35]$ but there are other occupational and dietary inducers. Variation in CYP1A2 activity was also related to polymorphisms in the CYP1A2 genes and consequently the effect of cigarette filter on the risk of bladder cancer development is dependent on this polymorphism. Further investigations on the role of CYP1A2 polymorphisms on the risk of bladder cancer performed on larger population of smokers are needed.

\section{CONCLUSIONS}

This epidemiological study suggests that the increased incidence of bladder cancer may be attributed to change in smoking habit associated to the substitution of plain cigarette to cigarette with filter while the effect of smoking duration cannot be excluded. As bladder carcinogens, such as aromatic amines, are mainly metabolised by CYP1A2 enzyme, a significantly higher CYP1A2 activity observed in bladder cancers patients when compared to control subjects suggests that high CYP1A2 activity could be a susceptible factor for bladder cancer in smokers. Changing in cigarette smoking habit associated to higher enzyme activity may be considered as a potential biomarker in the aetiology of bladder cancer and must be confirmed on a larger study.

Although filter is effective in reducing the amounts of PAHs and nicotine, it is less effective to retain volatile carcinogenic compounds such as TSNs and aromatic amines so that smokers are more exposed to these potent carcinogens. The users of cigarette with filter increase the smoking frequency and inhales more deeply, retaining smoke longer. This lead as a consequence to a higher deposition of carcinogens smokes in all vascular body regions. These carcinogens undergo biotransformation to give active carcinogens which are able to form ADN adducts initiating the first steps of cancer process. Increased use of cigarette with filter associated to the polymorphic CYP1A2 enzyme activity could therefore be a main cause in the bladder cancer increase.

It appears that cigarette with filter is not less dangerous than the plain cigarette as expected. The new smoking behaviour is involved in the increase incidence of 
many aggressive cancer forms such as AD of the lung and bladder cancer. In addition, the introduction of cigarette with filter lead to an increase over time in the number of cancers localization attributed to smoking [15, 36].

Finally, cigarette with filter and more generally smoking is today a major public health problem and smoking cessation remains the primary preventive way for tobacco related-cancers and pathologies.

\section{REFERENCES}

[1] Parkin, D.M., Pisani, P. and Ferlay, J. (1994) At least one of seven cases of cancer is caused by smoking. Global estimate for 1985. International Journal of Cancer, 59, 494-504. doi:10.1002/ijc.2910590411

[2] Pelluchi, C., Bosetti, C., Negri, E., Malvezzi, M. and La Vecchia, C. (2006) Mechanisms of disease: The epidemiology of bladder cancer. Nature Clinical Practice Urology, 3, 327-340. doi:10.1038/ncpuro0510

[3] Baris, D. et al. (2009) A case-control study of smoking and bladder cancer risk: Emergent patterns over time. Journal of the National Cancer Institute, 101, 1553-1561. doi:10.1093/jnci/djp361

[4] Boffetta, P. (2008) Tobacco smoking and risk of bladder cancer. Scandinavian Journal of Urology and Nephrology, 42, 45-54. doi:10.1080/03008880802283664

[5] Samanic, C., Kogevinas, M., Dosemeci, M., Malats, N., Real, F.X., Garcia-Closas, M., Serra, C., Carrato, A., García-Closas, R., Sala, M., Lloreta, J., Tardon, A., Rothman, N. and Silverman, D.T. (2006) Smoking and Bladder Cancer in Spain: Effects of Tobacco Type, Timing, Environmental Tobacco Smoke, and Gender. Cancer Epdemio Markers, 15, 1345-1354.

[6] Van Hemelrijck M. J. J., Michaud, D.S., Connolly, G.N. and Kabir, Z. (2009) Tobacco use and bladder cancer patterns in three western European countries. Journal of Public Health, 31, 335-344. doi:10.1093/pubmed/fdp062

[7] Raman, J.D., Messer, J., Sielatycki, J.A. and Hollenbeak, C.S. (2010) Incidence and survival of patients with carcinoma of the ureter and renal pelvis in the USA, British Journal of Urology International, 107, 1059-1064.

[8] Alberg, A.J. and Hebert, J.R. (2009) Cigarette Smoking and Bladder Cancer: A New Twist in an Old Saga? Journal of the National Cancer Institute, 101, 1525-1526. doi:10.1093/jnci/djp385

[9] López, A.G., González, C.A., Errezola, M., Escolar, A., Izarzugaza, I., Nebot, M. and Riboli, E. (1991) Tobacco smoke inhalation pattern, tobacco type, and bladder cancer in Spain. American Journal of Epidemiology, 134, 830-839.

[10] Hoffmann, D., Rivenson, A., Chung, F.L. and Wynder, E.L. (1993) Potential inhibitors of tobacco carcinogenesis. Annals of the New York Academy of Sciences, 28, 140-160. doi:10.1111/j.1749-6632.1993.tb39169.x

[11] Arnaud, M.J. (1993) Metabolism of caffeine and other components of coffee. In: Garattini, S. Ed., Caffeine, Coffee and Health, Raven Press, New York.

[12] B'chir, F., Laouani, A., Ksibi, S., Arnaud, M.J. and Saguem, S. (2007) Cigarette filter and the incidence of lung adenocarcinoma among Tunisian population. Lung can- cer Amsterdam Netherlands, 57, 26-33. doi:10.1016/j.lungcan.2007.01.034

[13] Lilienfeld, A.M., Levin, M. and Moore, G.E. (1956) The association of smoking with cancer of the urinary bladder in humans. Archives of Internal Medicine, 98, 129-135.

[14] U. S. Department of Health and Human Services. (2004) The Health Consequences of Smoking, A Report of the Surgeon General, Atlanta.

[15] Gandini, S. et al. (2008) Tobacco smoking and cancer: A meta-analysis. International Journal of Cancer, 122, 5-64. doi:10.1002/ijc.23033

[16] Gough, K.R., Read, A.E., McCarthy, C.F. and Waters, A.H. (1963) Megaloblastic anaemia due to nutritional deficiency of folic acid. QJM, 32, 243-256.

[17] Chanarin, I., MacGibbon, B.M., O’Sullivan, W.J. and Mollin, D.L. (1959) Folic-acid deficiency in pregnancy. The pathogenesis of megaloblastic anaemia of pregnancy. Lancet, 2, 634-639. doi:10.1016/S0140-6736(59)91409-6

[18] Pavanello, S., Mastrangelo, G., Placidi, D., Campagna, M., Pulliero, A., Carta, A., Arici, A. and Porru, S. (2010) CYP1A2 polymorphisms, occupational and environmental exposures and risk of bladder cancer. European Journal of Epidemiology, 25, 491-500. doi:10.1007/s10654-010-9479-8

[19] Van der Boon J. (2001) Low-tar filter cigarettes and an aggressive type of lung cancer. Lancet Oncology, 2, 255. doi:10.1016/S1470-2045(00)00310-7

[20] Hoffmann, D., Rivenson, A., Murphy, S.E., Chung, F.L., Amin, S. and Hecht, S.S. (1993) Cigarette smoking and adenocarcinoma of the lung: The relevance of nicotinederived $\mathrm{N}$-nitrosamines. Journal of Smoking Related Disorder, 4, 165-189.

[21] Wogan, G.N., Hecht, S.S., Felton, J.S., Conney, A.H. and Loeb, L.A. (2004) Environmental and chemical carcinogenesis. Seminars in Cancer Biology, 14, 473-486. doi:10.1016/j.semcancer.2004.06.010

[22] Morabia, A. and Wynder, E.L. (1991) Cigarette smoking and lung cancer cell types. Cancer, 68, 2074-2048. doi:10.1002/1097-0142(19911101)68:9<2074::AID-CNC R2820680939>3.0.CO;2-X

[23] Wald, N., Idle, M. and Smith, P.G. (1977) Carboxyhaemoglobin levels in smokers of filter and plain cigarettes. The Lancet, $\mathbf{1}, 110-112$. doi:10.1016/S0140-6736(77)91702-0

[24] Lubin, J.H., Alavanja, M.C.R., Caporaso, N., Brown, L.M., Brownson, R.C., Field, R.W., Garcia-Closas, M. Hartge, P., Hauptmann, M., Hayes, R.B., Kleinerman, R., Kogevinas M, Krewski D, Langholz B, Le tourneau E G, Lynch. C.F, Malats, N., Sandler, N.P., Schaffrath-Rosario, A., Schoenberg, J.B., Silverman, D.T., Wang, Z., Wichmann, H.E., Wilcox, H.B. and Zielinski, J.M. (2007) Cigarette smoking and cancer risk: Modeling total exposure and intensity. American Journal of Epidemiology, 166, 479-489. doi:10.1093/aje/kwm089

[25] Hoffmann, D., Brunnemann, K.D., Prokopczyk, B. and Djordjevic, M.V. (1994) Tobacco-specific $N$-nitrosamines and Areca-derived $N$-nitrosamines: Chemistry, biochemistry, carcinogenicity and relevance to humans. Journal of Toxicology and Environmental Health, 41, 1-52. doi:10.1080/15287399409531825

[26] Hecht, S.S., Isaacs, S. and Trushin, N. (1994) Lung tumor induction in $\mathrm{A} / \mathrm{J}$ mice by the tobacco smoke carcin- 
gens4-(methyl-nitrosamino)-1-(3-pyridyl)-1-butanone and benzo (a) pyrene: A potentially useful model for evaluation of chemopreventive agents. Carcinogenesis, 15, 2721-2725. doi:10.1093/carcin/15.12.2721

[27] Balansky, R., Ganchev, G., ltcheva, M., Steele, V.E., D’ Agostini1, F. and De Flora S. (2007) Potent carcinogenicity of cigarette smoke in mice exposed early in life. Carcinogenesis, 28, 2236-2243. doi:10.1093/carcin/bgm122

[28] Landi, M.T., Sinha, R., Lang, N.P. and Kadlubar, F.F. (1999) Human cytochrome P4501A2. In: Vineis, P., Malats, N., Lang, M., d’Errico, A., Caporaso, N., Cuzick, J. and Moffetta, P., Eds., Metabolic polymorphisms and susceptibility to cancer. IARC Scientific Publications, Lyon, 173-195.

[29] Vineis, P., Talaska, G., Malaveille, C., Bartsch, H., Martone, T., Sithisarankul, P. and Strickland, P. (1996) DNA adducts in urothelial cells: Relationship with biomarkers of exposure to arylamines and polycyclic aromatic hydrocarbons from tobacco smoke. International Journal of Cancer, 65, 314-316. doi:10.1002/(SICI)1097-0215(19960126)65:3<314::AID -IJC6>3.0.CO;2-2

[30] Bonser, G.M., Bradshaw, L., Clayson, D.B. and Jull, J.W. (1956) A further study of the carcinogenic properties of ortho hydroxy-amines and related compounds by bladder implantation in the mouse. British Journal of Cancer, 10, 539-546. doi:10.1038/bjc.1956.63

[31] Lee, S.W., Jang, I.J., Shin, S.G., Lee, K.H., Yim, D.S., Kim, S.W., Oh, S.J. and Lee, S.H. (1994) CYP1A2 activity as a risk factor for bladder cancer. Journal of Korean
Medical Science, 9, 482-489.

[32] Guengerich, F.P. and Shimada, T. (1991) Oxidation of toxic and carcinogenic chemicals by human cytochrome P-450 enzymes. Chemical Research in Toxicology, 4, 391-407. doi:10.1021/tx00022a001

[33] Kamataki, T., Fujita, K., Nakayama, K., Yamazaki, Y., Miyamoto, M. and Ariyoshi, N. (2002) Role of human cytochrome P450 (CYP) in the metabolic activation of nitrosamine derivatives: Application of genetically engineered Salmonella expressing human CYP. Drug Metabolism Reviews, 34, 667-676. doi:10.1081/DMR-120005668

[34] Landi, M.T., Zocchetti, C., Bernucci, M., Kadlubar, F.F., Tannenbaum, S., Skipper, P., Bartsch, H., Malaveille, C., shields, P., Caporaso, N. and Vinei, P. (1996) CytochromeP4501A2: Enzyme Induction and Genetic Control, Determining 4-aminobiphenyl-hemoglobin Adduct Levels. Cancer Epidemiology, Biomarkers \& Prevention, 5, 693-698.

[35] Le Marchand, L., Hankin, J.H., Wilkens, L.R., Pierce, L.M., Franke, A., Kolonel, L.N., Seifried, A., Custer, L.J., Chang, W., Lum, J.A. and Donlon, T. (2001) Combined Effects of Well-done Red Meat, Smoking, and Rapid $\mathrm{N}$-Acetyltransferase 2 and CYP1A2 Phenotypes in Increasing Colorectal Cancer Risk. Cancer Epidemiology, Biomarkers \& Prevention, 10, 1259-1266.

[36] Working group on the Evaluation of Carcinogenic Risks to Humans. (2004) Tobacco smoke and involuntary smoking. IARC Monographs on the Evaluation of Carcinogenic Risks to Humans, Lyon, 1-1438. 